\title{
Consumo de riesgo y perjudicial de alcohol. Prevalencia y métodos de detección en la práctica clínica.
}

\author{
Beatriz Rosón Hernández \\ Servicio de Medicina Interna. Hospital Universitario de Bellvitge. Barcelona.
}

El objetivo de este artículo es revisar el conocimiento y la evidencia científica actuales en la prevalencia del consumo perjudicial de alcohol en diferentes ámbitos y los métodos de detección de este consumo en la práctica clínica. Es importante recalcar que esta revisión está basada fundamentalmente en dos documentos a cuya lectura les remito. Estos documentos son el libro elaborado por el Ministerio de Sanidad y Consumo en 2008 "Prevención de los problemas derivados del alcohol. $1^{a}$ Conferencia de prevención y promoción de la salud en la práctica clínica en España" (1) y el libro elaborado por el Primary Health Care European Project on Alcohol (PHEPA) "Alcohol and Primary Health Care: Clinical Guidelines on Identification and Brief Interventions" (2).

\section{Prevalencia del consumo de riesgo y perjudicial de alcohol}

\section{Prevalencia de consumo de alcohol en Europa}

El alcohol aparece como el tercer factor de riesgo de morbimortalidad precoz en la Unión Europea (UE) sólo por detrás del tabaquismo y la hipertensión arterial, siendo más importante que la obesidad o la hipercolesterolemia. La UE es también el mayor productor, exportador e importador de bebidas alcohólicas. Aunque la tendencia del consumo medio ha tendido a disminuir en las últimas décadas de los 15 litros de alcohol puro por adulto y año a mediados de los años 1970s a los menos de 9 litros a inicios de los 2000, la

\section{Figura 1}

Consumo total de alcohol en países europeos, 2003. Consumo per cápita. Litros de alcohol puro. Fuente: World Health Organization. Regional Office for Europe. European Health for all database (HFA-DB

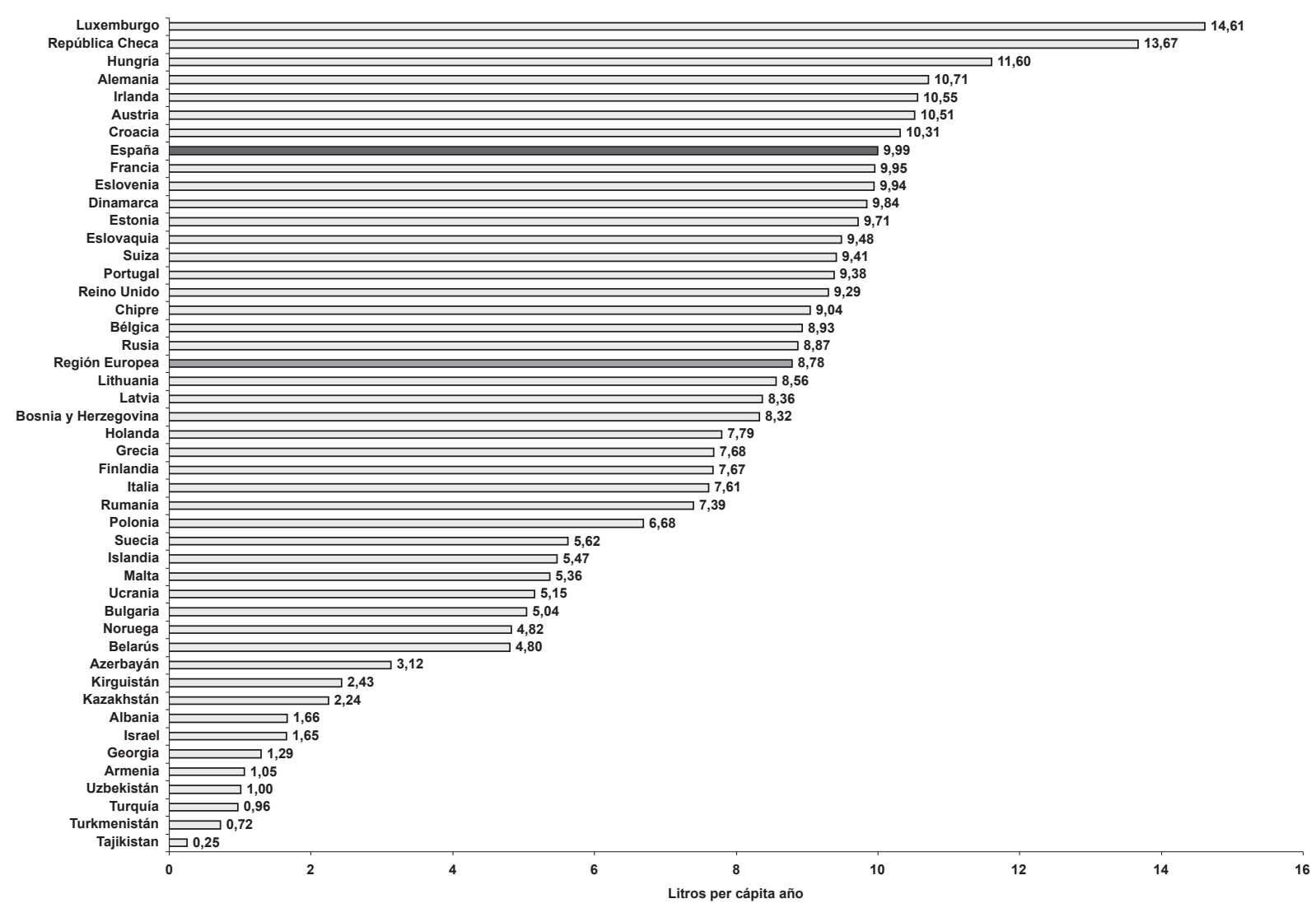


UE es la región del mundo con mayor consumo de alcohol per cápita. Estos niveles actuales están 2.5 veces por encima de la media del resto del mundo.

En la figura 1 se muestran las estimaciones más recientes de la Oficina Regional de la OMS para Europa en litros per cápita (3). Según estas estimaciones, España es el octavo país con mayor consumo de alcohol de toda la región europea.

Se calcula que en la UE 266 millones de adultos beben alcohol en los límites que se consideran de menor riesgo para la salud, hasta $20 \mathrm{~g}$ (mujeres) $040 \mathrm{~g}$ (hombres) por día. Se debe destacar que más de 58 millones de adultos (15\%) consumen por encima de este nivel, con 20 millones (6\%) de éstos que consumen más de $40 \mathrm{~g}$ (mujeres) $060 \mathrm{~g}$ (hombres) por día. Se estima que 23 millones de europeos/año tienen dependencia del alcohol. Cada año hasta 7 millones de adultos refieren haberse involucrado en peleas cuando bebían.

El consumo de riesgo y perjudicial de alcohol afecta a todos los grupos de edad, todos los estratos de la sociedad europea y a todos los países, aunque a aquellos con desventajas sociales les afectan de forma desproporcionada. Los jóvenes soportan de forma relativa una parte desproporcionada de esta carga ya que el alcohol origina el 25\% de la mortalidad en los hombres y del $10 \%$ en las mujeres (4). Diversos estudios muestran que un mayor nivel socioeconómico se relaciona con mayor frecuencia consumo, en particular para las mujeres. Los hombres con más educación tienen menos probabilidades de ser bebedores problema, en contraste con un efecto inverso encontrado en las mujeres. Se ha encontrado consistente evidencia que las personas con menor nivel socioeconómico se abstienen con mayor probabilidad de consumir alcohol. Sin embargo la intoxicación etílica y la dependencia del alcohol son más probables en aquellas personas con menor nivel socioeconómico, y esto ocurre en ambos sexos $(5,6)$.

Al daño físico y psicológico provocado en el propio consumidor, se debe añadir el daño producido en otras personas. El daño a terceros incluye el daño al feto en desarrollo, daño derivado de los accidentes de tráfico, violencia y crimen, el daño a las familias, niños, y el sufrimiento debido a la dependencia del alcohol.

El daño a terceros (perdidas intangibles) se ha estimado en 270.000 millones de euros anuales repartidos en dolor, sufrimiento y pérdida de vidas. El daño social (pérdidas tangibles) se estima en 125.000 millones de euros repartidos en gasto sanitario, crimen y pérdida de productividad (7).

Para definir el patrón de consumo en el ámbito europeo se han estudiado principalmente cuatro aspectos: el tipo de bebida alcohólica preferida, el contexto en que se consume, (principalmente el lugar y la relación con las comidas), la frecuencia de consumo de alcohol, y con qué frecuencia las bebidas se utilizan para la intoxicarse (a veces denominado «binge-drinking»).

Figura 2

Consumo per cápita de alcohol puro, según tipos de bebidas. España, 1982-2003.

Fuente: World Drink Trends 2005

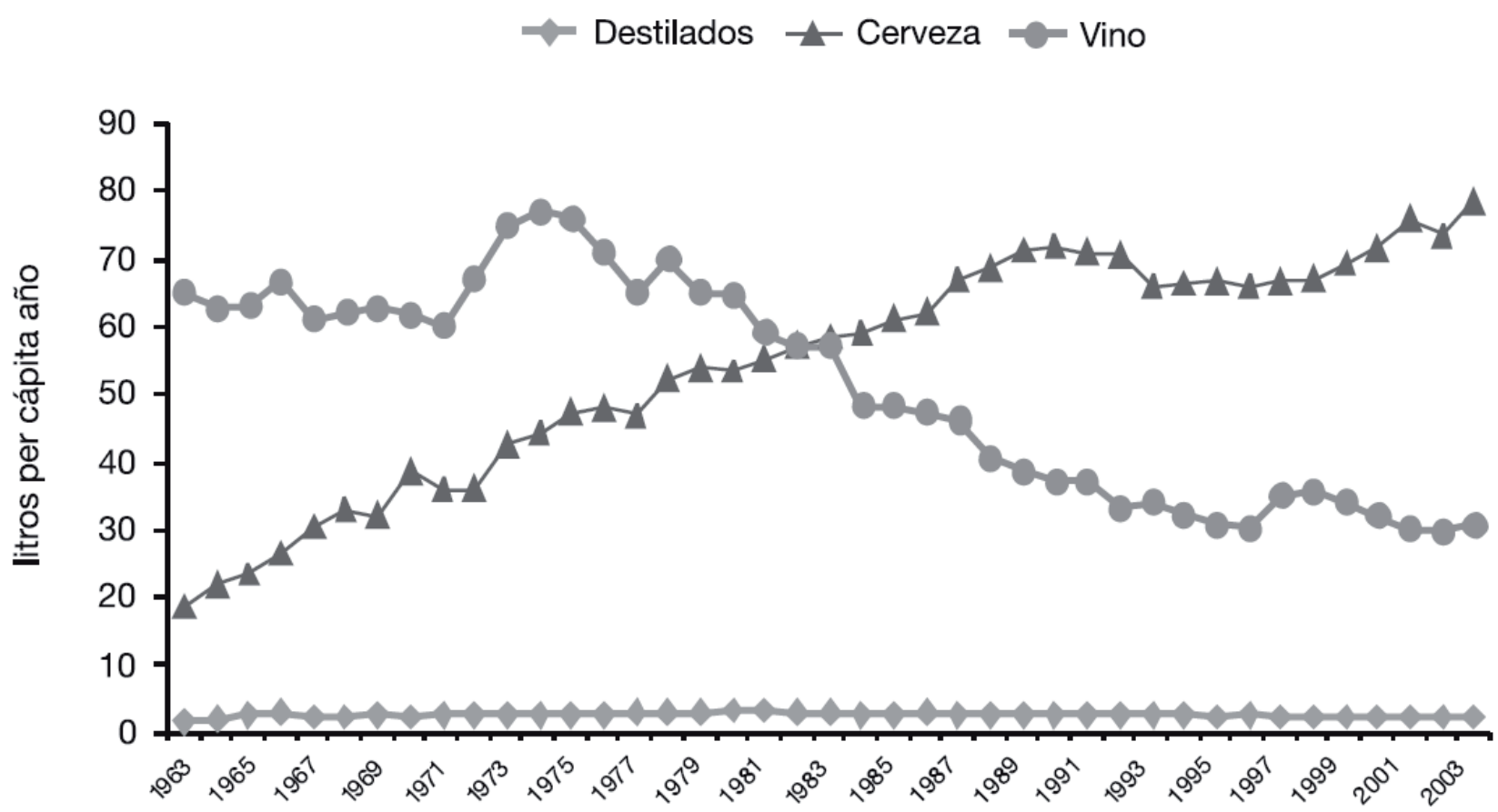


Cerca de la mitad del alcohol consumido en la UE se bebe en forma de cerveza (44\%), con el resto dividido entre el vino (34\%) y licores (23\%). Dentro de la UE15, en el norte y el centro se consume principalmente cerveza, mientras que los del sur de Europa, consumen principalmente vino (aunque España puede ser una excepción). Datos recientes muestran que en nuestro país se consume más cerveza que vino (figura 2). Además, en Grecia y España se bebe más alcohol procedente de las bebidas destiladas tradicionales locales que en los países del norte como Suecia y Noruega donde tradicionalmente se beben productos destilados (8).

En cuanto al contexto del consumo, los países europeos meridionales beben con más frecuencia durante las comidas que otros países de la UE15, sobre todo a la hora del almuerzo. Sin embargo, en Dinamarca y Suecia se produce un mayor consumo con las comidas que algunos países del sur de Europa, particularmente en España.

En general, la proporción de consumo total que se efectúa en la tarde/cena es similar en la mayoría de los países UE15 aunque, como en los países del sur de Europa se bebe mucho más a menudo en general, son más propensos a beber con cualquier comida.

Cuando se estudia la frecuencia de consumo de alcohol se observa que la gente en los países europeos meridionales

\section{Figura 3}

Distribución del binge drinking en la población europea según el Eurobarómetro 2003. Fuente: Eurobarometer 2003

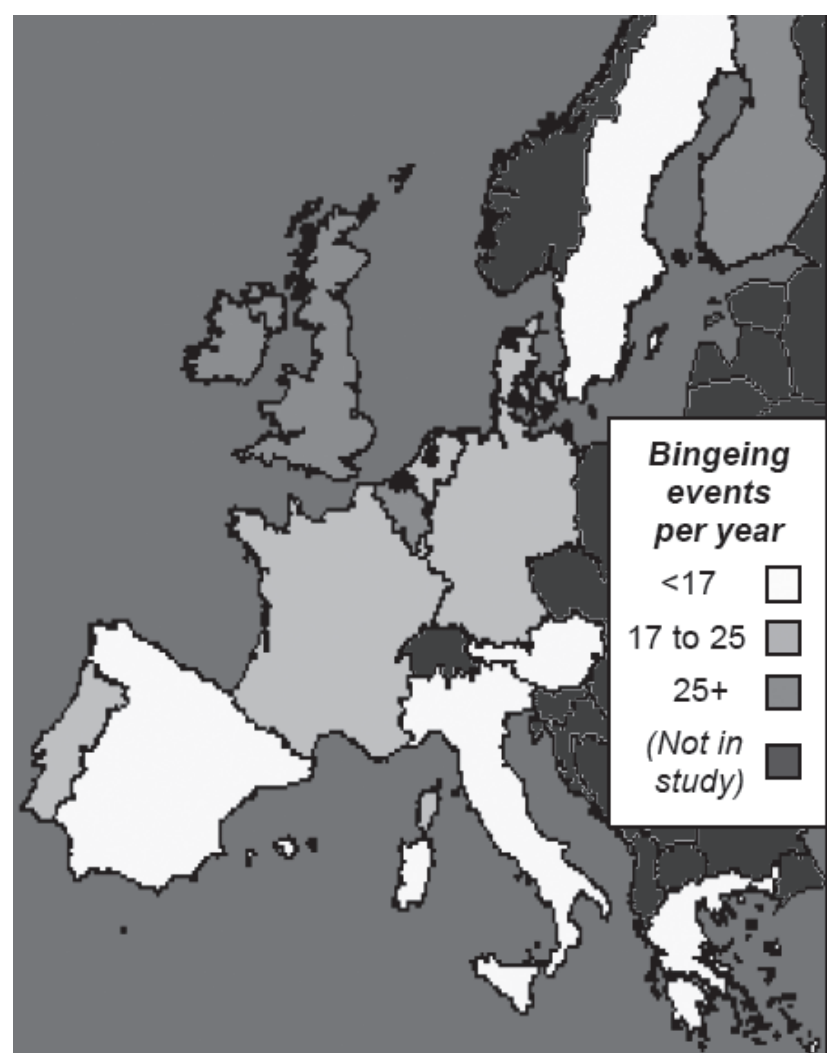

consume bebidas alcohólicas con más frecuencia que los que están en el norte de Europa, y tienen muchas más probabilidades de ser bebedores diarios. Esto se confirma según los datos del último Eurobarómetro publicado en 2007 en el que el $48 \%$ de los europeos que informaron de que habían consumido bebidas alcohólicas en los 30 días previos lo hicieron una vez por semana (25\%), $02-3$ veces por semana (23\%). Por otra parte, el $21 \%$ informaron de que habían consumido bebidas alcohólicas más de 4 días a la semana. Un análisis por países muestra que en 14 de los 25 Estados miembros la mayoría de los ciudadanos manifiesta haber bebido alcohol una vez a la semana en los 30 días previos. En los Estados bálticos, 36\% en Letonia, Lituania $35 \%$, Estonia el $29 \%$ y en Polonia el $28 \%$, la mayoría de los encuestados dijeron que habían bebido alcohol una sola vez durante el mes pasado. La mayoría de los bebedores portugueses consumían bebidas alcohólicas todos los días (47\%), pero - como veremos más adelante - normalmente sólo unas pocas bebidas por ocasión de consumo.

El "binge drinking" es una práctica frecuente en la UE (7). Se debe destacar que el término intoxicación no es sinónimo de binge drinking, aunque este último suela implicar un cierto nivel de intoxicación La terminología propuesta para este patrón de consumo en nuestro idioma es de consumo concentrado episódico, que consistiría en el consumo de 60 0 más gramos (6 UBEs), en varones, y de 400 más gramos (4 UBEs), en mujeres, concentrado en una sesión de consumo (habitualmente $4-6 \mathrm{~h}$ ), durante la que se mantiene un cierto nivel de intoxicación, es decir una alcoholemia no inferior a 0,8 $\mathrm{g} / \mathrm{L}(9)$.

Este patrón de consumo ha sido asociado con un considerable perjuicio social y una gran carga de morbilidad. Una revisión europea relaciona una gran variedad de factores socio-demográficos, individuales y sociales con el patrón de binge drinking. Este estudio mostró que los hombres tienden a realizar consumos episódicos abusivos con más frecuencia que las mujeres. Este patrón de consumo fue más prevalente entre los adolescentes y adultos jóvenes, y la prevalencia se fue estabilizando en años posteriores. Las condiciones socioeconómicas parecen tener también un efecto en el patrón de binge drinking (10).

En el Eurobarómetro publicado en marzo de 2007, la mayor parte de la población de la UE refería consumir 1 ó 2 consumiciones en una ocasión de consumo cuando consumían alcohol (59\%). Uno de cada diez europeos referían que tomaban habitualmente 50 más consumiciones por ocasión (11). Irlanda es con mucha diferencia el país en donde se realiza consumo concentrado episódico con mayor frecuencia, ya que el $36 \%$ de los encuestados referían beber 50 más consumiciones por ocasión de consumo. En el extremo opuesto se encuentran los portugueses (15\%) italianos (17\%) y luxemburgueses (17\%) que refieren que consumen una 0 menos unidades de bebida estándar (UBEs) por ocasión de consumo. 
Utilizando la definición de 5 o más UBEs por ocasión de consumo, el $11 \%$ de los varones bebedores en España referían consumos concentrados episódicos semanales frente a 2030\% de la UE-10 en el Eurobarómetro 2003. La figura 3 muestra la distribución del binge drinking en la población europea según el Eurobarómetro 2003 (12). La frecuencia de consumo episódico abusivo parece ser menor en Suecia que en muchos países del sur de Europa.

\section{Prevalencia del consumo de alcohol en España}

En la España actual, el alcohol es una parte de acontecimientos tan diversos como la comida diaria, servicios religiosos y las celebraciones. Su consumo está fuertemente arraigado en nuestra sociedad y existe gran tolerancia social sobre el uso de bebidas alcohólicas y las repercusiones que éste genera.

Además de los datos correspondientes al Eurobarómetro y al World Trends, disponemos en el ámbito nacional de los datos aportados por las Encuestas Domiciliarias sobre Drogas, dirigidas a población de 15-64 años. Estas encuestas son realizadas cada dos años por la Delegación del Gobierno para el Plan Nacional sobre Drogas del Ministerio de Sanidad y Consumo desde 1995. De hecho, en el 2003 un 76,6\% de la población de 15-64 años había consumido alcohol alguna vez en los últimos 12 meses, un $64,1 \%$ en los últimos 30 días, un $48,2 \%$ todas las semanas en los últimos 12 meses y un $14,1 \%$ diariamente en el mismo período. Datos preliminares de esta encuesta realizada en 2006 muestran que el $93.7 \%$ de la población española lo ha tomado "alguna vez". La mayoría lo consume de forma esporádica o habitual (76.7\% "en el último año"; 64.6\% "el último mes" y 14.9\% "a diario" durante el último mes). Según la última encuesta a escolares de 14-18 años publicada (Plan Nacional sobre Drogas 2004), el alcohol sigue siendo la sustancia más consumida entre los jóvenes de 14 a 18 años, Un 80,6\% declara haber consumido alcohol alguna vez, un $79,8 \%$ durante los doce meses previos a la entrevista y un $64 \%$ durante los treinta días previos (13).

En España, el vino y la cerveza están considerados todavía como alimentos y son una importante fuente de actividad económica. El vino genera un negocio de 4.800 millones de euros/año lo que representa un $0,65 \%$ del Producto Interior Bruto (PIB) español (4). El impacto económico real de los problemas derivados del consumo de alcohol en nuestro ámbito geográfico no ha sido todavía estimado correctamente. Según datos de 1996, se calcula que a partir de los costes directos (1.400 millones de euros) y de los costes indirectos por absentismo laboral y reducción de la eficiencia (2.400 millones de euros), el coste del daño producido por el alcohol en nuestro país es de alrededor de 3.800 millones de euros/año. En este valor no se incluyen sin embargo los costes derivados de la atención de consultas externas hospitalarias, las indemnizaciones por muerte e invalidez a causa de accidentes, los costes del síndrome alcohólico fetal, el de los años potenciales de vida perdidos, así como los años potenciales de vida productiva perdidos (14).

En cuanto al tipo de bebida preferida, según datos preliminares de la encuesta 2005-2006, las bebidas más consumidas en días laborables son el vino/cava. Un 10.4\% lo había bebido todos los días laborables, siendo el consumo mucho mayor entre los hombres. La cerveza/sidra son las más consumidas durante el fin de semana $(20,8 \%)$, seguida del vino/ cava $(15,9 \%)$ y los combinados/cubatas $(10,8 \%)$. El consumo de cerveza/sidra es mayor entre los hombres, siendo más frecuente entre los de 35 a 44 años (24.4\%) (15). Entre los jóvenes de 14-18 años, las bebidas predominantes son las de alta graduación, consumidas preferentemente en lugares públicos, con amigos y durante los fines de semana. Los lugares habituales de consumo son los bares, discotecas y la calle (16).

En España disponemos de escasa información acerca del consumo en forma de episodios de consumo concentrado 0 binge drinking. Datos recientes de una encuesta telefónica realizada a una muestra 12.037 personas representativa de la Comunidad de Madrid y con edades comprendidas 18 a 64 años muestran que la prevalencia de binge drinking fue de $14,4 \%$ en hombres y $6,5 \%$ en las mujeres. La prevalencia fue mayor en el grupo de edad más joven, con el más alto nivel educativo y con un alto promedio de consumo de alcohol. Sin embargo, 3 de cada 4 bebedores con este patrón mostró un consumo medio moderado. Durante cada episodio, los hombres consumían una media de $119 \mathrm{~g}$ del alcohol y las mujeres 83. El consumo de destilados representaba el $72 \%$ de total de consumo de alcohol con este patrón de consumo (17). En escolares de 15 a 16 años, este tipo de consumo se eleva al 35,5\%, siendo ligeramente más frecuente en los hombres que en las mujeres (38,8\% frente al 32,1\%). La tendencia describe desde 1997 un descenso moderado de la prevalencia de este indicador (4).

\section{Prevalencia del consumo en el ámbito sanitario}

Debido a los escasos estudios publicados, la prevalencia del consumo de riesgo y perjudicial de alcohol en el ámbito sanitario español no es bien conocida. En general se acepta que esta población está sobrerrepresentada en el ámbito sanitario. Un estudio multicéntrico realizado en el ámbito de la asistencia primaria en Cataluña demostró que sólo el $24,8 \%$ de las historias clínicas incluían consumo de bebidas alcohólicas en los últimos dos años. En este estudio, sólo 11 (2,8\%)- para todos los hombres- cumplían los criterios para el diagnóstico de consumo de riesgo. Esta cifra que es inferior a la de la población general puede explicarse por el bajo cribado realizado (18). Sin embargo, en otro estudio realizado en Albacete en el que se realizó una búsqueda prospectiva mediante el test de CAGE, se halló una prevalencia de dependencia del 17\% (19). 


\section{Deteccion del consumo de riesgo y perjudicial de alcohol en la práctica clínica}

El cribado de consumo de alcohol está ampliamente justificado en el ámbito sanitario. El alcohol está implicado de forma dosis-dependiente en una gran variedad de patologías físicas y mentales. El cribado de consumo de alcohol no sólo tiene sentido desde el punto de vista preventivo, sino también es de utilidad en el diagnóstico de la patología asociada tanto orgánica como psiquiátrica, para hacer el diagnóstico de la adicción y sentar la indicación de tratamiento profiláctico del síndrome de abstinencia alcohólica.

Muchos profesionales dicen realizar este cribado de forma sistemática. Spandorfer et al realizaron una encuesta por correo a internistas y médicos de cabecera en la que el $64,9 \%$ de los encuestados informó de que cribaban del $80 \%$ al $100 \%$ de sus pacientes para detectar abuso o dependencia del alcohol durante la visita inicial (20). Sin embargo, cuando se realizan revisiones de la historia clínica, los porcentajes de población cribados en la asistencia primaria son bajos 7-25\% en diferentes estudios realizados y los pacientes por lo general no recuerdan haber sido preguntados al respecto (18,21-24). En cuanto a la práctica hospitalaria, a excepción de la experiencia de Lloyd en la cual el alcohol se cuantificó en un 94\% de los expedientes médicos en una sala general de Medicina Interna,-aunque el autor puntualiza que con frecuencia los médicos subestimaban la cantidad consumida y pasaban por alto los síntomas de dependen- cia,- otros datos sobre la recogida o el cribado de consumo de alcohol son similares, a los hallados en la primaria, tanto en el ámbito internacional como el nacional (datos personales) (25-27).

Dado que muchos bebedores de riesgo pasan desapercibidos tras su contacto con el mundo sanitario, numerosos autores abogan por un abordaje sistemático para facilitar su identificación $(2,20,28)$. Si por razones de presión asistencial este no puede ser realizado de forma universal, el cribado del consumo de riesgo deberá realizarse sistemáticamente en los pacientes que presenten trastornos que puedan estar relacionados con el alcohol, tales como: hipertensión, cefaleas, dispepsia, ansiedad, depresión, irritabilidad, insomnio, déficits de atención, disfunciones sexuales, accidentes, enfermedad hepática, cáncer, resacas y bajo rendimiento laboral. Del mismo modo, se realizará el cribado sistemáticamente ante la presencia de signos clínicos como temblor, arañas vasculares, hepatomegalia, fétor enólico 0 elevaciones de VCM, GGT, AST o ALT $(2,28)$.

\section{Unidades de Bebida Estándar}

El cálculo del consumo de alcohol en gramos de alcohol puro sería la forma más exacta de cuantificar el consumo de alcohol. Para ello debemos aplicar una fórmula que consiste en multiplicar la cantidad de bebida en ml o cc por el número de grados de alcohol y por 0,8 , y este resultado se divide entre 100. El resultado nos permite conocer los gramos de alcohol de la consumición en cuestión. La anamnesis para

\section{Tabla 1}

Contenido de alcohol en gramos y equivalencia en Unidades de Bebida Standard (UBEs) e los productos consumidos con mayor frecuencia en España

\begin{tabular}{|l|c|c|c|c|}
\hline Bebida & $\begin{array}{c}\text { Volumen } \\
(\mathrm{cc})\end{array}$ & $\begin{array}{c}\text { Graduación } \\
\text { alcohólica }\end{array}$ & Gramos de alcohol & $\begin{array}{c}\text { Número de UBEs por } \\
\text { cada consumición }\end{array}$ \\
\hline Anís & 50 & 38 & 14.0 & 2 \\
\hline Cerveza & 200 & 6 & 9.6 & 1 \\
\hline Champán & 100 & 13 & 16.0 & 2 \\
\hline Cointreau & 50 & 40 & 16.0 & 1 \\
\hline Coñac & 50 & 40 & 16.0 & 2 \\
\hline Ginebra & 50 & 40 & 6.8 & 1 \\
\hline Jerez & 50 & 17 & 9.0 & 1 \\
\hline Orujo & 30 & 38 & 11.2 & 2 \\
\hline Pacharán & 50 & 28 & 11.2 & 2 \\
\hline Ponche & 50 & 28 & 16.8 & 2 \\
\hline Ron & 50 & 42 & 9.0 & 1 \\
\hline Vermut & 70 & 16 & 16.0 & 1 \\
\hline Vino & 100 & 42 & 12 & 2 \\
\hline Vodka & 50 & 45 & & 2 \\
\hline Whisky & 50 & 4.0 & & 2 \\
\hline
\end{tabular}

El valor de una UBE en España se establece en 10 gramos de alcohol 
averiguar exactamente los mililitros consumidos y la necesidad de tener un recordatorio con los grados de alcohol de cada bebida hacen que esta estrategia se haya reservado para estudios clínicos minuciosos, aunque sigue siendo el método de referencia en investigación científica.

La cuantificación del consumo en Unidades de Bebida Estándar (UBEs) permite una cuantificación rápida del consumo y su fácil conversión en gramos de alcohol puro. Resulta por tanto muy útil en la práctica clínica diaria. El valor de la UBE en España con una discreta diferencia Norte-Sur se establece en $10 \mathrm{~g}$ de alcohol y equivale a una consumición de vino (100ml), cava $(100 \mathrm{ml})$ o cerveza $(200 \mathrm{ml})$ y a media consumición de destilados o combinados $(25 \mathrm{ml})$ (29). La cuantificación del consumo en UBEs facilita el trabajo asistencial y sería actualmente el método de referencia en todos los niveles asistenciales (primaria, especializada y hospitalaria). Sin embargo, dada la variabilidad en gramos de alcohol en diferentes países, las UBEs han sido evitadas en los estudios epidemiológicos internacionales. De hecho, en EEUU y Canadá el valor de una UBE se acerca a los 14 gramos de alcohol puro, en Europa y Australia está en torno a los 10 gramos y en el Reino Unido se le asigna un valor de 8 gramos. La tabla 1 muestra las equivalencias equivalencia en UBEs de los productos consumidos con mayor frecuencia en España y la cuantificación exacta de gramos de alcohol puro.

\section{Niveles y categorías de consumo}

Tanto la OMS como la Comisión Europea se refieren a tres niveles de consumo en sus informes según la ingesta de gramos de alcohol al día, diferenciada según el sexo, considerando los abstemios como un cuarto nivel aparte. El nivel I se refiere a ingesta superior a 0 hasta40 $\mathrm{g}$ en hombres y $>0$ hasta $20 \mathrm{~g}$ en mujeres, nivel $\|>40$ hasta $80 \mathrm{~g}$ en hombres $0>20$ hasta $60 \mathrm{~g}$ en mujeres, y el nivel III $>80 \mathrm{~g}$ en hombres y $>60 \mathrm{~g}$ en mujeres (30-32). Esta clasificación, que es muy útil desde el punto de vista epidemiológico, puede llevar a confusión en la práctica clínica al asumir los niveles como ligero, moderado y excesivo. Se debe destacar que el nivel II se trata de un nivel de consumo de riesgo y no de un consumo "moderado".

\section{Patrones de consumo}

Para la valoración del patrón de consumo se debe realizar una historia clínica dirigida a evaluar el consumo de alcohol durante la historia del paciente, los síntomas de dependen- cia y la severidad de la abstinencia. Habitualmente, cómo se verá más adelante en los métodos de cribado, nos referiremos al consumo en los últimos 12 meses.

Se considera abstemios a aquellas personas que no han consumido alcohol en los últimos 12 meses. Esto puede no coincidir con la autodescripción de una persona como abstemia. Así, en nuestro medio, una persona que beba esporádicamente en acontecimientos sociales se puede definir a sí misma como abstemia mientras que en otras culturas se definiría como bebedor esporádico. Dentro de esta definición podrían encontrarse también aquellos pacientes con dependencia del alcohol que han abandonado el consumo. Se recomendaría desde el punto de vista clínico definir el patrón como dependencia en remisión.

Dado que el daño producido es, en general, directamente proporcional al nivel de consumo, no existe un nivel exento de riesgo. Se considera un consumo de riesgo como aquel en el cual no existen consecuencias actuales relacionadas con el consumo de alcohol pero sí riesgo futuro de daño psicológico, social y físico. Para la valoración del consumo de riesgo es importante registrar el consumo por días, si existen cambios de consumo particularmente durante el fin de semana y si existen consumos elevados por ocasión de consumo aunque sea de forma infrecuente. En ocasiones un consumo diario habitual bajo puede coexistir con un consumo semanal de riesgo o con una cantidad de riesgo en las ocasiones en que consume. En la tabla 2 se muestra la definición de consumo de riesgo teniendo en cuenta estos 3 parámetros En nuestro país, hemos adaptado la recomendación de la OMS y los niveles considerados de riesgo son para mujeres y personas mayores de 65 años $>17$ UBEs por semana 0 más de 3 UBEs por ocasión de consumo; para hombres >28 UBEs por semana 0 más de 5 UBEs por ocasión de consumo (33). Este último patrón se denomina binge drinking en la literatura anglosajona y se ha propuesto el término de consumo concentrado episódico como ha sido comentado previamente.

Se debe destacar que estos límites no se aplican a niños, adolescentes, mujeres embarazadas, personas que toman medicación que interacciona con el alcohol, personas que desarrollan actividades que requieran concentración, habilidad o coordinación (ej., conducción) 0 con comorbilidades que puedan verse afectadas por el consumo de alcohol (diabetes mellitus, hepatitis $\mathrm{C}$, etc.).

Tabla 2

Definición de consumo de riesgo en Europa

\begin{tabular}{|l|c|c|c|}
\hline & Consumo diario & Consumo semanal & Consumo por ocasión \\
\hline Mujeres y mayores de 65 años & $>2-2,5$ UBEs. & $>17$ UBEs & $\geq 4$ UBEs \\
\hline Hombres & $\geq 4$ UBEs & $>28$ UBEs & $\geq 6$ UBEs \\
\hline
\end{tabular}


Tabla 3

Interrogatorio sistemático sobre el consumo de alcohol (ISCA)

\begin{tabular}{|l|l|l|l|}
\hline & \multicolumn{1}{|c|}{ Cantidad (UBEs) } & Número de días & Total \\
\hline Laborables & & & \\
\hline Fines de semana & & & \\
\hline $\begin{array}{l}\text { ¿Si alguna vez consume bebidas alcohólicas (vino, cerveza, etc.), cuántas consumiciones hace en un día? (expresadas en UBEs) } \\
\text { ¿Con qué frecuencia? (número de días en una semana) } \\
\text { ¿Sus hábitos de consumo son diferentes durante los fines de semana (o en días laborables)? }\end{array}$ & \\
\hline
\end{tabular}

El consumo perjudicial o dañino de alcohol sería aquel patrón de consumo que causa daño a la salud. El daño puede ser físico ( $p$ ej. cirrosis hepática) o mental (depresión asociada al consumo). Las consecuencias sociales en sí mismas no son suficientes para el diagnóstico de consumo perjudicial 0 dañino.

La OMS en sus criterios de clasificación internacional de enfermedades (CIE-10 ó ICD-10) define la dependencia del alcohol como el conjunto de manifestaciones fisiológicas, de conducta y cognitivas que se desarrollan tras el consumo repetido de la sustancia y que típicamente incluyen un fuerte deseo de consumir, dificultad de controlar el uso (se consume más o durante más tiempo del deseado o existen intentos infructuosos de abandonar la sustancia), persistencia en el consumo a pesar de consecuencias negativas, reducción 0 abandono de actividades sociales, ocupacionales o recreativas para consumir o como consecuencia del consumo, aumento de la tolerancia, aparición de síntomas de abstinencia o consumo para evitar los síntomas de abstinencia (34).

En el lenguaje coloquial y a menudo también en el terreno profesional, se utilizan actualmente numerosos términos para describir el consumo de alcohol. Algunos de estos no tienen una definición claramente establecida o su significado puede ser confuso e incluso algunos son peyorativos. Estos términos deben evitarse por imprecisos y confusos o estigmatizantes. Entre ellos destacan los siguientes: alcoholismo, abuso de alcohol, consumo excesivo de alcohol, consumo moderado, consumo prudente, consumo social 0 consumo responsable (9).

\section{Métodos de cribado del consumo de riesgo y perjudicial de alcohol}

El consumo de riesgo se puede identificar preguntando directamente sobre el consumo o utilizando cuestionarios específicamente diseñados para este propósito. Existen además cuestionarios destinados al diagnóstico de la dependencia del alcohol. En el capítulo 5 del manual de la PHEPA encontrarán una amplia revisión de la evidencia científica en la utilización los diferentes instrumentos de cribado (2).

\section{Evaluación directa del consumo}

Existen dos métodos para registrar el consumo de alcohol referido por los pacientes: (1) las preguntas cantidad/frecuencia que piden al paciente que resuma las cantidades de alcohol consumidas y la frecuencia con la que lo beben durante un determinado periodo de tiempo (un día, una semana, un mes, etc.) 0 en términos de un patrón habitual 0 'típico'; y (2) estimaciones retrospectivas de las cantidades bebidas en un periodo de tiempo reciente, generalmente la última semana. Los métodos de estimación diaria consumen más tiempo y recursos y exigen un mayor esfuerzo al paciente. Los métodos de evaluación mediante ordenador 0 internet suponen ventajas en la conversión automática de la posibilidad de las respuestas de los pacientes en unidades de bebida estándar (UBE) sin que los resultados difieran sensiblemente de los que se obtienen con los métodos convencionales (35).

Las preguntas cantidad/frecuencia han demostrado su validez y utilidad. Por regla general los resultados son más objetivos cuando se circunscribe la exploración a un periodo concreto (una semana, un día, etc.) que cuando se usan términos más genéricos (habitualmente, un día normal, etc) (28). Las preguntas cantidad/frecuencia tienden a describir las conductas más habituales, más que el consumo promedio y existe evidencia de que los encuestados tienden a omitir los periodos de abstinencia cuando se les pregunta por los últimos 12 meses (36).

El Interrogatorio Sistematizado de Consumos Alcohólicos (ISCA) desarrollado por el grupo de Gual ha sido validado en España y ha mostrado ser un instrumento con mayor sensibilidad en hombres que en mujeres (70\%-81\% versus 50\%$73 \%$ y y con una elevada especificidad en ambos géneros (82\%-99\% en hombres y 97\%-100\% en mujeres). Como muestra la tabla 3, el ISCA consiste en preguntar directamente por las consumiciones en UBEs a diario o durante el fin de semana y ayuda a detectar consumos de riesgo de fin de semana. Ha sido comparado con el AUDIT y parece una herramienta de utilización fácil en asistencia primaria, puede ser utilizada indistintamente o de forma complementaria con el AUDIT (37).

\section{Tests de cribado}

Una revisión sistemática de 38 estudios apoya el uso de tests de screening en la asistencia primaria para detección de consumo perjudicial en adultos (rango de edades 3547 años) (38). Los instrumentos más aceptados y de mayor difusión para el diagnóstico de consumo de riesgo y perjudicial de alcohol y de dependencia alcohólica son el AUDIT, el AUDIT-C, el MALT y el CAGE. 


\section{Test AUDIT}

La prueba de la identificación de los trastornos por uso del alcohol (Alcohol Use Disorders Identification Test ó AUDIT) es la herramienta de detección mejor valorada y más utilizada, y también la recomendada por la OMS y el Ministerio de Sanidad y Consumo español para detectar estos trastornos.

EI AUDIT se desarrolló para detectar el consumo de riesgo y perjudicial de alcohol y particularmente para ayudar los clínicos a identificar aquellas personas que podrían beneficiarse de la reducción o abandono del consumo de alcohol.

El AUDIT consta de 10 preguntas sobre consecuencias de beber junto con preguntas sobre cantidad y frecuencia de consumo. Las tres primeras se refieren al consumo de riesgo de alcohol, las preguntas 4 a 6 son sobre síntomas de dependencia y las preguntas 7 a 10 sobre consumo perjudicial de alcohol (tablas 4 y 5). Cada pregunta puntúa de 0 a 4 , y la suma final permite identificar, en función del punto de corte, tanto los consumos de riesgo como la dependencia alcohólica. El tiempo de ejecución es de aproximadamente 5 minutos para realizar y contabilizar las preguntas. Cherpitel et al proponen los puntos de corte señalados en la tabla 4 para identificar consumo de riesgo, perjudicial y la dependencia (39).

Utilizando el punto de corte de 8, el AUDIT es una prueba sensible (51-97\%) para detectar uso perjudicial del alcohol y abuso o dependencia y puede ser utilizada sola 0 ser encajada en estudios más amplios del riesgo para la salud o de formas de vida. Su especificidad es del 78 al 96\% (33).

EI AUDIT ha sido estudiado en una gran variedad de países y culturas $(2,40)$, y es actualmente un instrumento internacional de cribado. A pesar de que su validez en mujeres es menor (41) el AUDIT se puede utilizar en ambos géneros. En la validación española de Rubio et al, el punto de corte para varones se estableció en 80 más y en mujeres en 60 más para la detección del consumo de riesgo. El efecto de la edad no se ha estudiado sistemáticamente pero en la validación española se propone el punto de corte para mayores de 60 años en 5 (42). Un estudio más reciente realizado en nuestro país no encuentra puntos de corte distintos entre mayores y menores de 65 años (43).

\section{Test AUDIT-C}

El AUDIT-C consta de las tres primeras preguntas del AUDIT. Ha sido utilizado y validado a nuestro medio en hombres y mujeres y se ha mostrado como una herramienta con una efectividad similar a la forma completa para la detección de consumo de riesgo de alcohol (44-45). En los diversos estudios el AUDIT-C ha mostrado una sensibilidad entre 54 y $98 \%$ y una especificidad entre el 57 y el $93 \%$ (38). En el estudio de validación español, se comparó el AUDIT-C con el AUDIT en pacientes con el diagnóstico de consumo de riesgo realizado por médicos de atención primaria. Las correlaciones entre los resultados del AUDIT-C, el AUDIT y el consumo alcohólico medido en UBEs fueron positivas y muy significativas. El AUDIT-C y el mostró sensibilidad y es- pecificidades similar al AUDIT en la detección de consumo de riesgo. Para los hombres el mejor punto de corte fue 5 (sensibilidad 92.4\%; especificidad 74.3\%), y entre las mujeres el mejor punto de corte fue 4 (sensibilidad $90.9 \%$ y especificidad 68.4\%) (44).

\section{Cuestionario CAGE}

El Test CAGE (Chronic Alcoholism General Evaluation; ) es un sencillo cuestionario de cuatro preguntas que permite detectar el alcoholismo(46) y existe una versión validada en castellano. Es un test de cribaje para la detección pero el diagnóstico del alcoholismo debería basarse en otras pruebas y no tiene una adecuada sensibilidad para detectar consumo de riesgo. Sin embargo, su uso se ha extendido gracias a su brevedad y facilidad de puntuación. De cualquier modo, Spandorfer refiere que el 95\% de médicos de cabecera e internistas encuestados contestaron que "a menudo" o "siempre" realizaban el interrogatorio de consumos mediante preguntas la cantidad/frecuencia para detectar el abuso del alcohol, pero sólo el 35\% contestaron que "frecuentemente" o "siempre" utilizaban el cuestionario CAGE (20).

Las preguntas del CAGE pueden administrarse solas o "camufladas" en el contexto de una entrevista más amplia, para evitar el posible rechazo.

Las preguntas son las siguientes:

1. ¿Ha tenido usted alguna vez la impresión de que debería beber menos?

2. ¿Le ha molestado alguna vez que la gente critique su forma de beber?

3. ¿Se ha sentido alguna vez mal o culpable por su costumbre de beber?

4. ¿Alguna vez lo primero que ha hecho por la mañana ha sido beber para calmar sus nervios o para librarse de una resaca?

Se considera positivo si se responde afirmativamente a un mínimo de dos preguntas. Su sensibilidad oscila entre 49 y $100 \%$ y su especificidad entre 79 y 100\%, en función del punto de corte propuesto y de la gravedad del problema relacionado con el alcohol que se esté valorando. Por regla general se recomienda limitar el uso del CAGE a la detección de la dependencia alcohólica, dado que el AUDIT tiene un mejor rendimiento para la detección de bebedores de riesgo (28).

\section{MALT}

El MALT (Munchner Alkoholismus Test) es un cuestionario muy utilizado para el diagnóstico de dependencia alcohólica y particularmente útil en aquellos casos con dependencia encubierta. Consta de dos partes, el MALT objetivo (MALT-0), que es cumplimentado por el entrevistador y que recoge datos de la anamnesis, exploración física y laboratorio, y el MALT subjetivo (MALT-S), que es autoadministrado y consta de 27 ítems que exploran aspectos psicoconductuales y repercusiones del consumo de alcohol. El test ha sido 
Tabla 4

Test AUDIT y puntos de corte

\begin{tabular}{|c|c|c|}
\hline Items & Criterios operativos de valoración & \\
\hline 1.¿Con qué frecuencia consume alguna bebida alcohólica? & $\begin{array}{l}\text { 0.Nunca } \\
\text { 1.Una o menos veces al mes } \\
\text { 3.De } 2 \text { a } 3 \text { veces a la semana }\end{array}$ & $\begin{array}{l}\text { 2.De } 2 \text { a } 4 \text { veces al mes } \\
4 \text { Cuatro o más veces a la semana }\end{array}$ \\
\hline $\begin{array}{l}\text { 2. ¿Cuántas consumiciones de bebidas alcohólicas suele reali- } \\
\text { zar en un día de consumo normal? }\end{array}$ & $\begin{array}{l}\text { 0.Una ó } 2 \\
\text { 1.Tres ó } 4 \\
\text { 3. De } 7 \text { a } 9\end{array}$ & $\begin{array}{l}\text { 2.Cinco ó } 6 \\
\text { 4. Diez o más }\end{array}$ \\
\hline $\begin{array}{l}\text { 3. ¿Con qué frecuencia toma } 6 \text { ó más bebidas alcohólicas en } \\
\text { una sola ocasión de consumo? }\end{array}$ & $\begin{array}{l}\text { 0.Nunca } \\
\text { 1.Menos de una vez al mes } \\
\text { 3.Semanalmente }\end{array}$ & $\begin{array}{l}\text { 2.Mensualmente } \\
\text { 4.A diario } 0 \text { casi a diario }\end{array}$ \\
\hline $\begin{array}{l}\text { 4.¿Con qué frecuencia en el curso del año ha sido incapaz de } \\
\text { parar de beber una vez había empezado? }\end{array}$ & $\begin{array}{l}\text { 0.Nunca } \\
\text { 1.Menos de una vez al mes } \\
\text { 3.Semanalmente }\end{array}$ & $\begin{array}{l}\text { 2.Mensualmente } \\
\text { 4.A diario o casi a diario }\end{array}$ \\
\hline $\begin{array}{l}\text { 5. ¿Con qué frecuencia en el curso del último año no pudo } \\
\text { hacer lo que se esperaba de usted porque había bebido? }\end{array}$ & $\begin{array}{l}\text { 0.Nunca } \\
\text { 1.Menos de una vez al mes } \\
\text { 3.Semanalmente }\end{array}$ & $\begin{array}{l}\text { 2.Mensualmente } \\
\text { 4.A diario o casi a diario }\end{array}$ \\
\hline $\begin{array}{l}\text { 6. ¿Con qué frecuencia en el curso del último año ha necesita- } \\
\text { do beber en ayunas para recuperarse después de haber bebido } \\
\text { mucho el día anterior? }\end{array}$ & $\begin{array}{l}\text { 0.Nunca } \\
\text { 1.Menos de una vez al mes } \\
\text { 3.Semanalmente }\end{array}$ & $\begin{array}{l}\text { 2.Mensualmente } \\
\text { 4.A diario } 0 \text { casi a diario }\end{array}$ \\
\hline $\begin{array}{l}\text { 7. ¿Con qué frecuencia en el curso del último año ha tenido } \\
\text { remordimientos o sentimientos de culpa después de haber be- } \\
\text { bido? }\end{array}$ & $\begin{array}{l}\text { 0.Nunca } \\
\text { 1.Menos de una vez al mes } \\
\text { 3.Semanalmente }\end{array}$ & $\begin{array}{l}\text { 2.Mensualmente } \\
\text { 4.A diario o casi a diario }\end{array}$ \\
\hline $\begin{array}{l}\text { 8. ¿Con qué frecuencia en el curso del último año no ha podido } \\
\text { recordar lo que sucedió la noche anterior porque había estado } \\
\text { bebiendo? }\end{array}$ & $\begin{array}{l}\text { 0.Nunca } \\
\text { 1.Menos de una vez al mes } \\
\text { 3.Semanalmente }\end{array}$ & $\begin{array}{l}\text { 2.Mensualmente } \\
\text { 4.A diario } 0 \text { casi a diario }\end{array}$ \\
\hline $\begin{array}{l}\text { 9. ¿Usted o alguna otra persona han resultado heridos porque } \\
\text { usted había bebido? }\end{array}$ & $\begin{array}{l}\text { 0.No } \\
\text { 2.Sí, pero no en el curso del último año }\end{array}$ & 4.Sí, el último año \\
\hline $\begin{array}{l}\text { 10. ¿ Algún familiar, amigo, médico o profesional sanitario han } \\
\text { mostrado preocupación por su consumo de bebidas alcohólicas } \\
\text { o le han sugerido que deje de beber? }\end{array}$ & $\begin{array}{l}\text { 0.No } \\
\text { 2.Sí, pero no en el curso del último año }\end{array}$ & 4.Sí, el último año \\
\hline
\end{tabular}

Puntos de corte

Sin problemas: Hombres; 0-7; Mujeres: 0-5 / Bebedor de riesgo: Hombres: 8-12; Mujeres:6-12

Problemas físico - psíquicos y probable dependencia alcohólica: Hombres: 13-40; Mujeres: 13-40.

Tabla 5

Dominios y ítems del AUDIT

\begin{tabular}{|l|c|l|}
\hline \multirow{4}{*}{ Dominios } & Numero de pregunta & Contenido del ítem \\
\hline \multirow{3}{*}{ Consumo de riesgo } & 1 & Frecuencia de consumo \\
\cline { 2 - 3 } & 2 & Cantidad típica \\
\cline { 2 - 3 } Síntomas de dependencia & 3 & Frecuencia de consumo elevado (binge drinking) \\
\cline { 2 - 3 } & 4 & Perdida de control sobre el consumo \\
\cline { 2 - 3 } & 5 & Aumento de la relevancia del consumo \\
\hline \multirow{3}{*}{ Consumo perjudicial de alcohol } & 6 & Consumo matutino \\
\cline { 2 - 3 } & 7 & Sentimiento de culpa frente al consume \\
\cline { 2 - 3 } & 8 & Lagunas de memoria \\
\cline { 2 - 3 } & 9 & Lesiones relacionadas con el consumo \\
\cline { 2 - 3 } & 10 & Otros se preocupan por el consumo \\
\hline
\end{tabular}


Tabla 6

Test MALT. Versión validada en castellano.

\section{Cuestionario MALT-0}

1. Enfermedad hepática (mínimo 1 síntoma clínico, p.e., hepatomegalía, dolor a la presión, etc, y al menos 1 valor de laboratorio patológico, p.e., GOT,GPT,GGT).(Sólo procede cuando se tratade una hepatopatía alcohólica o de origen desconocido: descartar hapatitis vírica, hepatomegalía de hepatopatía congestiva, etc.)

2. Polineuropatía (sólo procede cuando no existen otras causas conocidas, p.e.,diabetes mellitus o intoxicaciones crónicas específicas)

3. Delirium tremens (actual o en la anamnesis)

4. Consumo de alcohol superior a los $150 \mathrm{ml}$ (en la mujer 120ml) de alcohol puro al día, al menos durante unos meses

5. Consumo de alcohol superior a los 300ml (en la mujer $240 \mathrm{ml}$ ) de alcohol puro, una o más veces al mes

6. Aliento alcohólico (en el momento de la exploración)

7. Los familiares o allegados ya han buscado, en una ocasión, consejo acerca del problema alcohólico del paciente (al médico, asistente social, 0 instituciones pertinentes)

\begin{tabular}{|l|l|}
\hline SI & N0 \\
\hline & \\
\hline & \\
\hline & \\
\hline & \\
\hline
\end{tabular}

\section{Cuestionario MALT-S}

1. En los últimos tiempos me tiemblan a menudo las manos

2. A temporadas, sobre todo por las mañanas, tengo una sensación nauseosa o ganas de vomitar

3. Alguna vez, he intentado calmar la resasca, el temblor, o la náusea matutina con alcohol

4. Actualmente, me siento amargado por mis problemas y dificultades

5. No es raro que beba alcohol antes del desayuno o del almuerzo

6. Tras los primeros vasos de una bebida alcohólica, a veces siento la necesidad irresistible de seguir bebiendo

7. A menudo pienso en el alcohol

8. A veces he bebido alcohol, incluso cuando el médico me lo ha prohibido

9. En las temporadas en que bebo más, como menos

10. En el trabajo me han llamado ya la atención por mis ingestas de alcohol o alguna vez he faltado al trabajo por haber bebido demasiado la víspera

11. Ultimamente, prefiero beber alcohol a solas ( $\mathrm{y}$ sin que me vean)

12. Bebo de un trago y más deprisa que los demás

13. Desde que bebo más, soy menos activo

14. A menudo me remuerde la conciencia (sentimiento de culpa) después de haber bebido

15. He ensayado un sistema para beber (p.e., no beber antes de determinadas horas)

16. Creo que debería limitar mis ingestas

17. Sin alcohol no tendría yo tantos problemas

18. Cuando estoy excitado bebo alcohol para calmarme

19 Creo que el alcohol está destruyendo mi vida

20. Tan pronto quiero dejar de beber como cambio de idea y vuelvo a pensar que no

21. Otras personas no pueden comprender porque bebo

22. Si yo no bebiera me llevaría mejor con mi esposa/o ( o pareja)

23. Ya he probado a pasar temporadas sin alcohol

24. Si no bebiera, estaría contento conmigo mismo

25. Repetidamente me han mencionado mi "aliento alcohólico"

26. Aguanto cantidades importantes de alcohol sin apenas notarlo

27. A veces, al despertar, después de un día de haber bebido mucho, aunque sin embriagarme no recuerdo en absoluto las cosas que ocurrieron la víspera

* Todas las respuestas negativas puntúan 0, la respuesta afirmativa del MALT-0: 4 puntos, y la respuesta del MALT-S:1 punto 
validado en nuestro país (47). Cada respuesta positiva del MALT-0 suma 4 puntos, mientras cada respuesta positiva del MALT-S suma un punto.

Se considera que puntuaciones iguales 0 superiores a 11 son diagnósticas de alcoholismo y entre 6 y 10 sospecha de alcoholismo o de riesgo alcohólico. Su sensibilidad es del $100 \%$ y su especificidad del $80 \%$ (48).

\section{Evaluación de marcadores biológicos de enolismo}

Los marcadores bioquímicos del consumo alcohólico son los enzimas hepáticos (-glutamil transferasa (GGT) y las aminotransferasas aspartatoaminotranferasa (AST) y alaninaminotransferasa (ALT), la transferrina deficiente en carbohidratos o desialotransferrina (TDC) y el volumen corpuscular medio (VCM). Los marcadores biológicos no detectan consumos de $40 \mathrm{~g}$ a $60 \mathrm{~g} / \mathrm{día}$ que pueden producir el daño en otras áreas sociofamiliares o psicopatológicas. No existen en la actualidad marcadores fiables para detectar el consumo excesivo de alcohol, especialmente en pacientes jóvenes y con uso discontinuo del alcohol (49-50). Aunque actualmente no disponemos de un marcador biológico con una especificidad y sensibilidad suficientes, el uso de una batería de marcadores puede ser útil en el abordaje de los pacientes que niegan o minimizan el consumo, para determinar la afectación física del consumo y para monitorizar la evolución.

\section{Conclusiones}

En resumen, el consumo de alcohol es altamente prevalente en nuestro país aunque la prevalencia de consumo de riesgo y perjudicial de alcohol en el ámbito sanitario no es bien conocida. El daño producido por el alcohol es en general directamente proporcional al nivel de consumo y no existe por lo tanto un nivel exento de riesgo. Los términos recomendados para referirnos al consumo de alcohol son consumo de riesgo, consumo perjudicial y dependencia. La cuantificación del consumo en UBEs es actualmente el método de referencia en la práctica clínica, ya que permite una cuantificación rápida del consumo y su fácil conversión en gramos de alcohol puro (multiplicar número de UBEs por 10). El AUDIT-C es el cuestionario de elección para el cribado de consumos de riesgo en el ámbito sanitario. El AUDIT completo es el cuestionario de elección para la detección del síndrome de dependencia alcohólica. Los marcadores biológicos no deben ser utilizados como instrumentos de cribado, aunque pueden ser útiles en el manejo clínico de los pacientes.

\section{BIBLIOGRAFIA}

1. Prevención de los problemas derivados del alcohol. $1^{\text {a }}$ Conferencia de prevención y promoción de la salud en la práctica clínica en España. 2008. Ministerio de Sanidad y consumo.

2. Anderson P, Gual A, Colom J. (2005) Alcohol and Primary Health Care: Clinical Guidelines on Identification and Brief Interventions. Departament de Salut. Generalitat de Catalunya. Barcelona.]

3. World Health Organization Regional Office for Europe. European health for all database (HFA-DB). Updated: June 2006. Disponible en: http://data.euro.who.int/hfadb/

4. Galán I, Alvarez J. Capítulo 1 Magnitud del problema. En Prevención de los problemas derivados del alcohol. $1^{\text {a }}$ Conferencia de prevención y promoción de la salud en la práctica clínica en España. 2008. Ministerio de Sanidad y consumo.

5. Jacobsen BK. Frequency of alcohol use and the level of education. J Intern Med. 1989 Jun;225(6):417-22.

6. Marques-Vidal P, Dias CM. Trends and determinants of alcohol consumption in Portugal: results from the national health surveys 1995 to 1996 and 1998 to 1999. Alcohol Clin Exp Res. 2005 Jan;29(1):89-97.

7. Anderson P, Baumberg B. (2006) Alcohol in Europe. London: Institute of alcohol studies.

8. World Drink trends 2005. World Advertising Research Council; 2005.

9. Rodriguez Martos A, Rosón B. Capítulo 2. Definición y terminología. En : Prevención de los problemas derivados del alcohol. $1^{\text {a }}$ Conferencia de prevención y promoción de la salud en la práctica clínica en España. 2008. Ministerio de Sanidad y consumo.

10. Kuntsche E, Rehm J, Gmel G. Characteristics of binge drinkers in Europe. Soc Sci Med 2004; 59:113-27

11. Special Eurobarometer. Attitudes towards Alcohol. 2007. En: http://ec.europa.eu/ health/ph_determinants/life_style/alcohol/documents/ebs272_en.pdf

12. Eurobarometer Health, Food and Alcohol and Safety. 2003. En: http://ec.europa.eu/ health/ph_determinants/life_style/alcohol/documents/ebs_186_en.pdf

13. Plan Nacional sobre Drogas. Encuesta Domiciliaria (EDADES) 2003. Disponible en: http://www.pnsd.msc.es/Categoria2/observa/pdf/domiciliaria2003.pdf

14. García-Sempere A, Portella E. Los estudios del coste del alcoholismo: marco conceptual, limitaciones y resultados en España. Adicciones 2002; 14(Supl 1):141-53

15. Plan Nacional sobre Drogas. Encuesta Domiciliaria (EDADES) 2005-2006. Disponible en:http://www.pnsd.msc.es/Categoria2/observa/pdf/Domiciliaria2005-2006.pdf

16. Plan Nacional sobre Drogas. Encuesta estatal sobre uso de drogas en enseñanza secundaria 2004. Disponible en: http://www.pnsd.msc.es/Categoria2/observa/pdf/ escolar2004.pdf

17. Valencia-Martín JL, Galán I, Rodríguez-Artalejo F. Binge drinking in Madrid, Spain. Alcohol Clin Exp Res. 2007;31:1723-30.

18. Bueno Ortiz JM, Aubà Llambrich J, Altaba Barceló A et al. Un estudio A multicenter primary care study on alcohol consumption. Aten Primaria. 1997; 19(2):80-83.

19. Escobar F, Espí F, Canteras M. Problems related to alcohol consumption in primary health care: prevalence and clinical profile. Fam Pract. 1993;10(4):424-30.

20. Spandorfer JM, Israel Y, Turner BJ. Primary care physicians' views on screening and management of alcohol abuse: inconsistencies with national guidelines. J Fam Pract. 1999 Nov;48(11):899-902.

21. Brotons C, Iglesias M, Martin-Zurro A, Martin-Rabadan M, Gene J. Evaluation of preventive and health promotion activities in 166 primary care practices in Spain. The Coordinating Group For Prevention and Health Promotion in Primary Care in Spain. Fam Pract. 1996;13:144-51

22. Aalto M, Seppä K. Usefulness, length and content of alcohol-related discussions in primary health care: the exit poll survey. Alcohol Alcohol. 2004;39:532-5.

23. Rush BR, Urbanoski KA, Allen BA. Physicians' enquiries into their patients' alcohol use: public views and recalled experiences.Addiction. 2003; 98:895-900.

24. Aalto M, Pekuri P, Seppa K. Primary health care professionals' activity in intervening in patients' alcohol drinking: a patient perspective. Drug Alcohol Depend. $2002 \mathrm{Mar}$ $1 ; 66: 39-43$.

25. Hearne R, Connolly A, Sheehan J. Alcohol abuse: prevalence and detection in a general hospital. J R Soc Med. 2002;95(2):84-7.

26. Wu SI, Liu SI, Fang CK, Hsu CC, Sun YW. Prevalence and detection of alcohol use disorders among general hospital inpatients in eastern Taiwan. Gen Hosp Psychiatry. 2006;28(1):48-54.

27. Chen $\mathrm{CH}$, Chen WJ, Cheng AT. Prevalence and identification of alcohol use disorders among nonpsychiatric inpatients in one general hospital.Gen Hosp Psychiatry. 2004;26(3):219-25.

28. Gual A. Capítulo 3: Cribado del consumo de alcohol. En Prevención de los problemas derivados del alcohol. $1^{\text {a }}$ Conferencia de prevención y promoción de la salud en la práctica clínica en España. 2008. Ministerio de Sanidad y consumo

29. Rodriguez-Martos A, Gual A, Llopis Llacer JJ. La Unidad de Bebida estándar: un registro simplificado del consumo de bebidas alcohólicas. Med Clin (Barc ) 1999; 112(12):446-450.

30. World Health Organisation. Lexicon of alcohol and drug terms published by the World Health Organization. 2006. En: http://www.who.int/substance_abuse/terminology/ who_lexicon/en/ 
31. Rehm J, Room R,Monteriro M, Gmel G, Graham K, Rehn T, Sempos CT, Frick U, Jerningan D. Alcohol use. En: Ezzati M, López AD, Rodgers A, Murray CJL. Comparative quantification of health risks; Global and regional burden of disease attributable to selected major risk factors. Vol 1. Geneva, World Health Organization, 2004 (pp: 959-1108).

32. European Commission Report: Alcohol in Europe. 2006 En: http://ec.europa.eu/ health-eu/news_alcoholineurope_en.htm

33. Babor TF, Higgins-Biddle J, Saunders J, Monteiro MG. AUDIT: The Alcohol Use Disorders Identification Test. Guidelines for Use in Primary Care. 2nd ed. World Health Organization, 2001.

34. International Statistical Classification of Diseases and Related Health Problems 10th Revision. Version for 2007. En: http://www.who.int/classifications/apps/icd/ icd10online/

35. Miller, E. T., Neal, D. J., Roberts, L. J., Baer, J. S., Cressler, S. O., Metrik, J. , Marlatt, $G$. A. Test-retest reliability of alcohol measures: is there a difference between internet-based and traditional methods? Psychology of Addictive Behaviors. 2002;16: 56-63.

36. Weisner, C., Kaskutas, L. A., Hilton, M. E. \& Barile, A. L. (1999) 'When you were drinking' vs. 'in the past 12 months': the impact of using different time frames in clinical and general populations. Addiction, 94, 731-736.

37. Gual A, Contel M, Segura L, Riba A, Colom J. (2001) El ISCA (Interrogatorio Sistematizado de Consumos Alcohólicos), un nuevo instrumento para la identificación precoz de bebedores de riesgo. Medicina Clínica (Barc) 117: 685-689.

38. Fiellin DA, Reid MC, O'Connor PG. Screening for alcohol problems in primary care: a systematic review. Arch Intern Med. 2000;160(13):1977-1989.

39. Cherpitel, C.J. Analysis of cut points for screening instruments for alcohol problems in the emergency room. Journal of Studies on Alcohol 1995;56:695-700.

40. Wu SI, Huang HC, Liu SI, Huang CR, Sun FJ, Chang TY, Shih SC, Jeng KS. Validation and Comparison of Alcohol-Screening Instruments for Identifying Hazardous Drinking in Hospitalized Patients in Taiwan. Alcohol Alcohol. 2008 . 2008 May 8. [Epub ahead of print]
41. Steinbauer, J.R., Cantor, S.B., Holder, C.E. and Volk, R.J. Ethnic and sex bias in primary care screening tests for alcohol use disorders. Annals of Internal Medicine 1998;29:353-362

42. Rubio Valladolid G, Bermejo Vicedo J, Caballero Sanchez-Serrano MC, Santo-Domingo Carrasco J. Validación de la Prueba para la Identificación de Transtornos por Uso de Alcohol (AUDIT) en Atención Primaria. Revista Clínica Española; 1998:198:1114.

43. Gómez A, Conde A, Santana JM, Jorrín A, Serrano IM, Medina R. The diagnostic usefulness of AUDIT and AUDIT-C for detecting hazardous drinkers in the elderly. Aging Ment Health. 2006;10:558-61

44. Gual A, Segura L, Contel M et al. Audit-3 and audit-4: effectiveness of two short forms of the alcohol use disorders identification test. Alcohol Alcohol. 2002;37(6):591596.

45. Gómez A, Conde A, Santana JM, Jorrín A. Diagnostic usefulness of brief versions of Alcohol Use Disorders Identification Test (AUDIT) for detecting hazardous drinkers in primary care settings. J Stud Alcohol. 2005;66:305-8..

46. Ewing JA. Detecting alcoholism: the CAGE questionnaire. JAMA 1984; 252: 19051907.

47. Rodriguez Martos A, Suárez R. MALT (Munchner Alkoholismus Test): validación de la versión española para el diagnóstico de alcoholismo. Rev Psquiatr Psicol Med 1984; 16:421-32.

48. Guardia G, Jiménez-Arriero MA, Pascual P, Flórez G, Contel M.. Guía clínica de alcoholismo. Socidrogalcohol.2007. En: http://socidrogalcohol.psiquiatria.com/ documentos/200705alcoholismo.pdf

49. Salaspuro, M. (1999) Carbohydrate-deficient transferrin as compared to other markers of alcoholism: a systematic review. Alcohol, 19, 261-271.

50. Sharpe, P. C. (2001) Biochemical detection and monitoring of alcohol abuse and abstinence. Annals of Clinical Biochemistry, 38, 652-664. 